\title{
To be or not to be: Mechanical versus biological prosthesis at the pulmonary valvular position
}

\author{
Guo-Wei He, MD, PhD, DSc
}

\footnotetext{
From the Department of Cardiovascular Surgery, TEDA International Cardiovascular Hospital, Chinese Academy of Medical Sciences and Peking Union Medical College, Tianjin and Zhejiang University and Hangzhou Normal University, Hangzhou, China; and Department of Surgery, Oregon Health and Science University, Portland, Ore.

Disclosures: Author has nothing to disclose with regard to commercial support.

Received for publication May 30, 2017; accepted for publication June 5, 2017; available ahead of print July 1, 2017.

Address for reprints: Guo-Wei He, MD, PhD, DSc, TEDA International Cardiovascular Hospital, Tianjin China; and Clinical Professor of Surgery, Oregon Health and Science University, Portland, OR (E-mail: gwhezj@ 163. com).

J Thorac Cardiovasc Surg 2017;154:1379-80

$0022-5223 / \$ 36.00$

Copyright (C) 2017 by The American Association for Thoracic Surgery

http://dx.doi.org/10.1016/j.jtcvs.2017.06.012
}

Heart valve replacement has been standard therapy for valves that cannot be repaired; however, the use of mechanical versus biological prostheses has long been a subject of debate. In general, mechanical prostheses are used in younger patients for their durability, and biological prostheses are used for their biological nature without the longterm need for anticoagulants. The more complex issue regards the use of valve prostheses in the right heart-the tricuspid and pulmonary valves. ${ }^{1,2}$ Owing to the low pressure, thrombosis is a problem, and thus the use of biological prostheses in the pulmonary position is preferred in many centers. The durability of bioprosthetic valves in the pulmonary position is suboptimal, however. Stable valve function is maintained only until approximately 5 years after implantation on average; by 10 years, roughly $80 \%$ will require reoperation or manifest valve dysfunction. ${ }^{1}$ This problem is particularly important in the pulmonary valve position, given the general younger age of those patients compared with patients requiring valve replacement in the mitral or aortic position. In this sense, mechanical prostheses are superior for valve replacement in the pulmonary position.

In their article in this issue of the Journal, Pragt and colleagues ${ }^{3}$ report on the medium-to long-term performance of mechanical valves in the pulmonary position. Surprisingly, their results show a limited risk of valvular thrombosis, with promising results for thrombolytic treatment. The authors retrospectively collected and analyzed data on 364 patients with mechanical valves implanted in the pulmonary position between 1965 and 2014 from multiple centers. As reported, the median duration of follow-up was 4.3 years (range, 0-27 years), and the mean age at implantation was $27.2 \pm 12.2$ years. Tetralogy of Fallot was the most common primary cardiac diagnosis $(69.8 \%)$. The freedom from valvular thrombosis was $91 \%$ at 5 years and $86 \%$ at 10 years after pulmonary valve replacement.

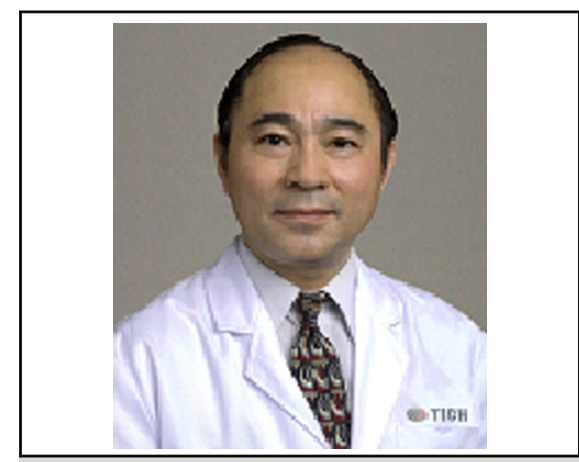

Guo-Wei He, MD, PhD, DSc

\section{Central Message}

Evaluation of the medium- to long-term performance of mechanical valves in the pulmonary position indicates a limited risk of valvular thrombosis, with promising results for thrombolytic treatment. A randomized prospective study is needed to compare the results between mechanical valves and bioprostheses at the pulmonary position.

See Article page 1371.

In the patients who developed thrombosis, thrombolysis had a success rate of $88 \%$. The freedom from reoperation was $97 \%$ at 5 years and $91 \%$ at 10 years. These results clearly indicate that the use of mechanical valve prostheses in the pulmonary valve position is a viable option, particularly after tetralogy of Fallot repair. Considering the fact that mean age at implantation was 27 years, the benefit from mechanical valve prosthesis implantation can be even more significant.

These results are encouraging for the future use of mechanical valve prostheses in the pulmonary position. A few points should be emphasized, however. First, this is a retrospective study, and a more specific and randomized prospective study is needed. Second, the study has a long time frame, which makes the patient cohort somehow heterogeneous. Third, and most important, the authors do not compare mechanical valve prostheses and bioprostheses in the pulmonary position, and thus no definitive conclusions can be drawn from this study as to which type is favored in the pulmonary position. A randomized prospective study is needed to compare these 2 types of prostheses. Finally, an important question regarding anticoagulant therapy is bleeding 
complications. Achieving a balance between the risk of thrombosis and the risk of bleeding after valve replacement is an art, particularly with the use of mechanical prostheses. The target international normalized ratio (INR) ranged on the higher side in this study, reflecting the authors' concerns about thrombosis when a mechanical prosthesis is placed in the low-pressure right heart system. With a target INR ranging from 2.5 to 4.5 (their Table 1), it would not be possible to have zero complications of bleeding, including cerebral complications. Unfortunately, the authors did not report bleeding complications, and only mentioned that 1 death occurred due to pulmonary hemorrhage with an INR $>7$. A more comprehensive study of this point is needed.

\section{References}

1. Lee C, Park CS, Lee CH, Kwak JG, Kim SJ, Shim WS, et al. Durability of bioprosthetic valves in the pulmonary position: long-term follow-up of 181 implants in patients with congenital heart disease. J Thorac Cardiovasc Surg. 2011;142:351-8.

2. Li ZX, Guo ZP, Liu XC, Kong XR, Jing WB, Chen TN, et al. Surgical treatment of tricuspid regurgitation after mitral valve surgery: a retrospective study in China. $J$ Cardiothorac Surg. 2012;7:30.

3. Pragt H, van Melle JP, Javadikasgari H, Seo DM, Stulak JM, Knez I, et al. Mechanical valves in the pulmonary position: an international retrospective analysis. $J$ Thorac Cardiovasc Surg. 2017;154:1371-8.e1. 